\title{
Multiple liquid-liquid critical points and density anomaly in core-softened potentials
}

\author{
Marco Aurélio A. Barbosa, ${ }^{1, *}$ Evy Salcedo, ${ }^{2, \dagger}$ and Marcia C. Barbosa ${ }^{3, \dagger}$ \\ ${ }^{1}$ Programa de Pós-Graduacão em Ciência de Materiais, Faculdade UnB Planaltina, Universidade de Brasília, Planaltina-DF, Brazil \\ ${ }^{2}$ Departamento de Física, Universidade Federal do Santa Catarina, Florianópolis-SC, Brazil \\ ${ }^{3}$ Instituto de Física, Universidade Federal do Rio Grande do Sul, Porto Alegre-RS, Brazil
}

(Received 19 June 2012; revised manuscript received 4 December 2012; published 11 March 2013)

\begin{abstract}
The relation between liquid-liquid phase transitions and waterlike density anomalies in core-softened potentials of fluids was investigated in an exactly solvable one-dimensional lattice model and in a three-dimensional fluid with a Fermi-like potential, the latter by molecular dynamics. Both systems were shown to present three liquid phases, two liquid-liquid phase transitions closely connected to two distinct regions of anomalous density increase. We propose that an oscillatory behavior observed on the thermal expansion coefficient as a function of pressure can be used as a signature of the connection between the liquid-liquid phase transition and density anomaly.
\end{abstract}

DOI: 10.1103/PhysRevE.87.032303

PACS number(s): 61.20.Gy, 65.20.-w

\section{INTRODUCTION}

The phase behavior of single component systems as particles interacting via the so-called core-softened (CS) potentials has received attention since the pioneering work of Stell and Hemmer proposing the possibility of a second critical point in addition to the usual liquid-gas critical point [1]. These potentials exhibit a repulsive core with a softening region with a shoulder or a ramp [1-3] which are analytically and computationally tractable while being capable of retaining the qualitative features of the real fluid systems. Furthermore, Debenedetti and collaborators [4], using thermodynamic arguments, showed that the isobaric thermal expansion coefficient $(\alpha)$ of these potentials might have an anomalous negative value and consequently a region where density increases with temperature. Since for high temperatures the density decreases with temperature, these potentials can exhibit a temperature of maximum density (TMD) connecting the two regions.

The thermodynamic anomalies predicted by these models occur in liquids such as Te [5], Ga, Bi [6], S [7,8], liquid water [9], and $\mathrm{Ge}_{15} \mathrm{Te}_{85}$ [10], and were found in simulations for silica [11,12], silicon [13], and $\mathrm{BeF}_{2}$ [11]. In addition, experiments in phosphorous indicate the presence of a liquid-liquid phase transition [14] and similar transitions were observed by simulations in models of silica [15], silicon [13], and liquid water [16].

In the particular case of water, the hypothesis of the existence of two liquid phases has been indirectly supported by experimental results in confined systems [17]. In spite of the limit of $235 \mathrm{~K}$ below which water cannot be found in the liquid phase without crystallization, two amorphous phases, a low density amorphous phase and a high density amorphous phase, were observed at much lower temperatures and their relation to a metastable liquid-liquid phase transition was argued [18]. More recently a third amorphous phase, the very high density amorphous phase, has been observed [19], which suggests the possibility of the existence of a very high density liquid phase.

\footnotetext{
*aureliobarbosa@unb.br

†esalcedo@fsc.ufsc.br

${ }^{\ddagger}$ marcia.barbosa@ufrgs.br
}

Therefore the issue of a liquid-liquid phase transition and its connection with the existence of a region in the pressuretemperature phase diagram where the density decreases with a decrease of temperature is itself an interesting topic. It is accepted that the presence of two accessible length scales in the potential allow for the system to have two liquid phases and a density anomaly. These two structures can arise from the competition between the two length scales in the potential $[3,4]$. Two liquid and amorphous structures can also appear in core-softened one length scale potentials, such as the Gaussian model [20] and the Hertzian sphere model [21], or a very soft ramp model [22]. In both examples the scales would be visible by the double peak at the radial distribution function. In the case of a single scale, the two peaks are associated with the range of the core-softened potential as defined by Debenedetti et al. [4]. In the Gaussian and Hertzian models the Debenedetti et al. [4] condition applies only for a very narrow range of distances. Consequently the density anomaly appears in a very small region in the pressure-temperature phase diagram [20,21].

The accessibility is the ingredient that explains why a density anomaly derived in one dimension (1D) does not necessarily hold at higher dimensions and why a density anomaly derived for a smooth potential might be lost if the slope linking the two length scales becomes infinite [23-25]. In addition, it is expected that in systems in which a density anomalous region is present, the diffusion coefficient, the excess entropy, and the structural order parameter would also present an unusual behavior [26-28], with a certain hierarchy that will depend on the detail of the potential [26,29].

In this paper we show, by means of an exactly solvable 1D model and numerical simulations of a similar threedimensional (3D) potential, that a three length scale potential might exhibit three critical points and two density anomalous regions if the different length scales would be accessible. Previous computer simulations of core-softened potentials with three scales proved that these models can exhibit three critical points [30] but we find that two liquid-liquid phase transitions are shown to occur that are associated with two temperature of maximum density lines.

In addition, we propose a way to identify if a liquid-liquid critical point would be present by exploring the behavior of $\alpha$ for temperatures above the critical temperature. We show 


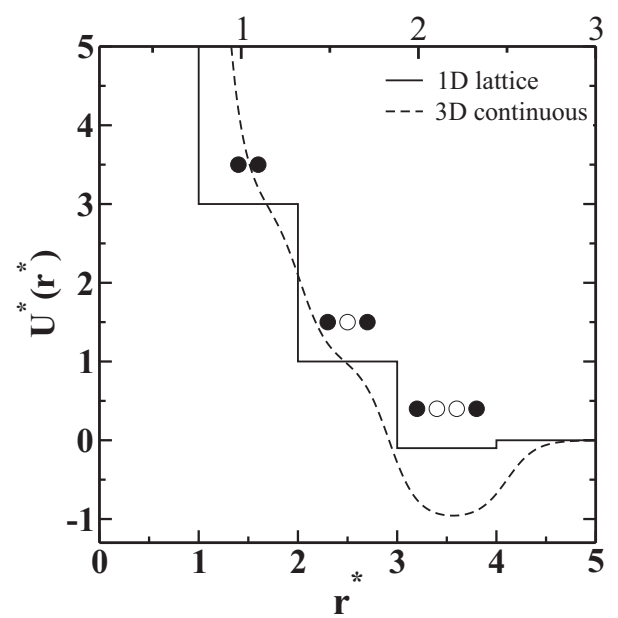

FIG. 1. Representation of the pair potentials investigated in this paper: a one-dimensional lattice fluid and an isotropic threedimensional continuous core-softened model with three scales of interaction. The top (bottom) horizontal axis is used to represent the continuous (lattice) system.

that, for systems in which there is a density anomalous region close to criticality, $\alpha$ exhibits a peculiar behavior diverging to $+\infty$ for pressures above the critical pressure and to $-\infty$ for pressures below the critical pressure.

\section{THE ONE-DIMENSIONAL MODEL}

The core-softened potentials we analyze are illustrated in Fig. 1 in units of length $\sigma$ and of energy $\epsilon$. They are composed by a hard core of diameter $\sigma$, two repulsive shoulders, and an attractive well with depth $-\lambda \epsilon$, where we employ $\lambda=1 / 10$ for the $1 \mathrm{D}$ case and $\lambda=1$ for the $3 \mathrm{D}$ case. Both potentials are detailed below.

We first investigate the 1D to obtain analytical insights and to become familiar with the properties that will also appear in the 3D case. The linear lattice has length $L$ and $N$ sites separated by a distance $\sigma=L / N$. The exact form of the Gibbs free energy derived in the framework of the Takahashi method [31] is given by

$$
G(T, P, N)=-N k_{B} T \ln \left[\sum_{r=1}^{\infty} e^{-\beta h(r ; P)}\right],
$$

where $\beta=1 /\left(k_{B} T\right)$ with $k_{B}$ the Boltzmann constant, and $h(r ; P)=U(r)+P r$ the microscopic pair enthalpy, with $U(r)$ being the interaction energy between neighbor molecules. Expression (1) is calculated using $U(\sigma)=3 \epsilon, U(2 \sigma)=\epsilon$, $U(3 \sigma)=-\epsilon / 10$, and $U(r)=0$ for $r>3 \sigma$.

The minimization of Eq. (1) in the ground state results in three configurations depicted in Fig. 1: a low density liquid (LDL) phase, a high density liquid (HDL) phase, and a very high density liquid (VHDL) phase, besides a gas phase (G) (not illustrated). The coexistence between these phases allows for three ground state phase transitions (GSTPs) to occur at reduced pressures $\left(P^{*}=P \sigma / \epsilon\right) P_{\mathrm{G}-\mathrm{LDL}}^{*}=0, P_{\mathrm{LDL}-\mathrm{HDL}}^{*}=1.1$, and $P_{\mathrm{HDL}-\mathrm{VHDL}}^{*}=2.0$. In what follows the temperature is reduced as $T^{*}=k_{B} T / \epsilon$.

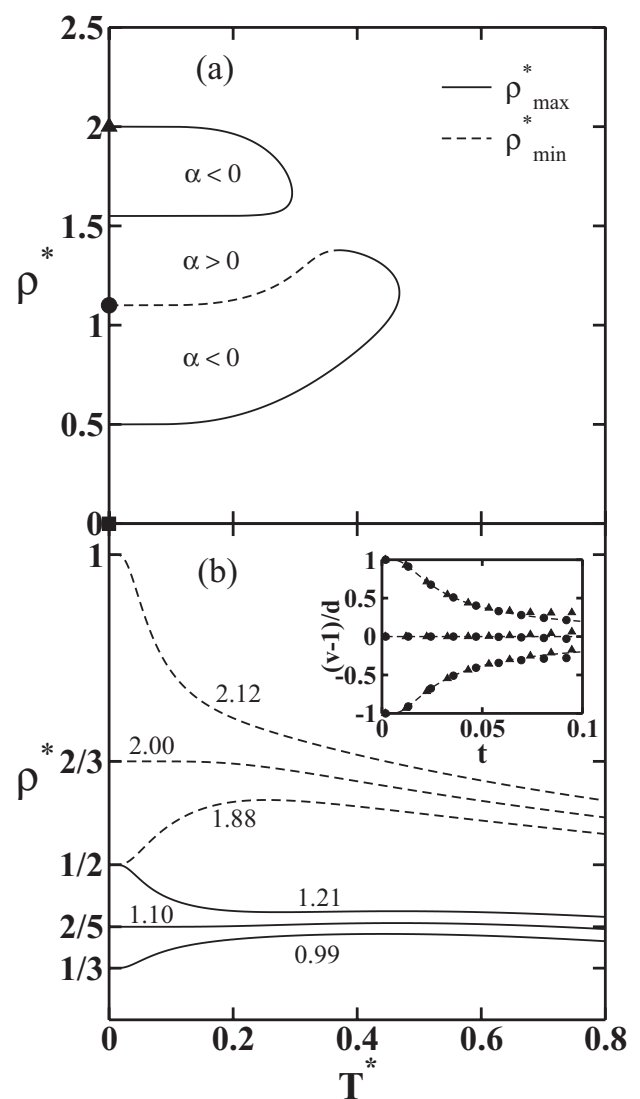

FIG. 2. (a) Pressure vs temperature phase diagram for the 1D case. Continuous and dotted lines indicate the TmD and the TMD lines, while symbols locate the ground state phase transitions. (b) Density as a function of temperature, for various pressures. The inset shows the reduced volume as a function of the reduced temperature near the two critical points, according to (3).

The full phase diagram for the 1D case is illustrated on Fig. 2(a) with the symbols indicating the three GSPTs described above. From the LDL-HDL critical point (circle) emerges a line of temperature of minimum density (TmD) followed by a line of temperature of maximum density (TMD), while from the HDL-VHDL critical (triangle) point there appears a TMD. These lines separate the region where the thermal expansion coefficient is positive from the regions where $\alpha$ is negative. Figure 2(b) illustrates the density as a function of the temperature for various pressures, some of them crossing the TMD and the TmD lines.

Further comprehension of the relation between the density anomaly and the ground state phase transitions can be gained by visualizing $\alpha$ as a function of pressure (at fixed temperature), as shown in Fig. 3. In the usual systems, $\alpha \rightarrow \infty$ as the critical point is approached from any path in the pressure-temperature plane. The peculiarity of models in which the criticality is associated with the density anomaly is that, below the critical pressure $P_{c}$, the sign of $\alpha$ is negative, while for $P>P_{c}$ its sign is positive. Consequently, $\alpha$ displays an oscillatory behavior as a function of pressure, with $\alpha\left(P \rightarrow P_{c}^{-}\right) \rightarrow-\infty$ and $\alpha\left(P \rightarrow P_{c}^{+}\right) \rightarrow+\infty$, which is a signature of the connection between the TMD line (or TmD) and the critical point [32]. 


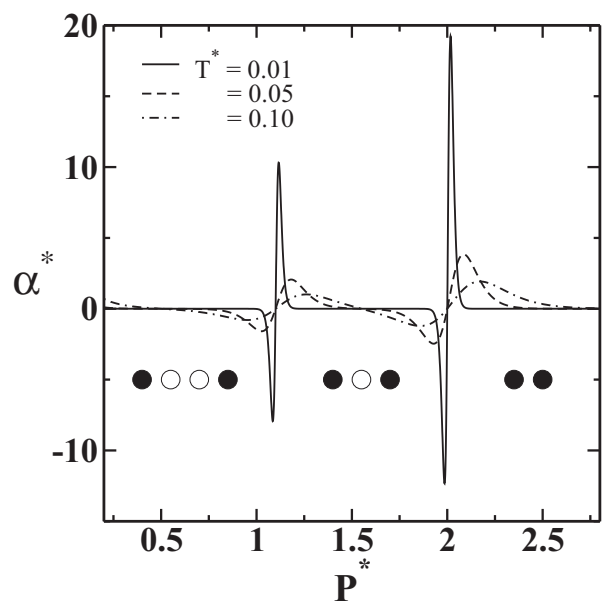

FIG. 3. Thermal expansion coefficient $\left(\alpha^{*}=\epsilon \alpha\right)$ as a function of pressure at fixed temperatures, for the 1D case.

The relation between criticality and anomalous density behavior can be rationalized by looking at the thermodynamic close to the GSPT within the approximation adopted in Ref. [33]. For a lattice model with a core-softened interaction the reduced Gibbs free energy per particle in the vicinity of any GSPT can be written in terms $r_{1}$ and $r_{2}$, the average distances between the particles in the two coexisting phases, leading to

$$
g(t, p)=h_{c}+p-t \ln \left[2 \cosh \left(\frac{p d}{t}\right)\right],
$$

where $g=G /\left(N P_{c} r_{c}\right), \quad t=k_{B} T / P_{c} r_{c}, \quad p=\left(P-P_{c}\right) / P_{c}$, and $h_{c}=h\left(r_{1}\right) /\left(P_{c} r_{c}\right)$. For the LDL-HDL transition discussed above, $r_{1}=2 \sigma, r_{2}=3 \sigma, d=\left(r_{1}+r_{2}\right) /\left(r_{2}-r_{1}\right)=$ $1 / 5$, and $r_{c}=\left(r_{1}+r_{2}\right) / 2=5 \sigma / 2$. It follows from (2) that the reduced volume per particle, $v=V /\left(N r_{c}\right)$, can be written as

$$
v(t, p)=1-d \tanh \left(\frac{p d}{t}\right) .
$$

In order to understand the relation between waterlike anomalies and phase transitions we investigate the behavior of molecular volume while approaching the GSPT, $t=p=0$. Close to this point the value of the reduced volume depends on the path along which criticality is approached, namely,

$$
\begin{aligned}
& \lim _{p \rightarrow 0} \lim _{t \rightarrow 0} v= \begin{cases}1-d, & p \rightarrow 0^{+}, \\
1+d, & p \rightarrow 0^{-},\end{cases} \\
& \lim _{t \rightarrow 0} \lim _{p \rightarrow 0} v=1 .
\end{aligned}
$$

The behavior of the reduced volume indicates that slightly below and above the LDL-HDL critical pressure the system is arranged according the LDL and the HDL, respectively, while exactly at the critical pressure the system becomes a mixture of the two phases as the temperature is reduced. Thus, the entropy gained by mixing with the HDL phase explains the density increase observed on the LDL side.

\section{THE THREE-DIMENSIONAL MODEL}

Next, we consider if the presence of three critical points and two regions of density anomaly observed for one dimension holds for three dimensions. To this end, we perform molecular
TABLE I. Coefficients of the core-softened pair potential of Eq. (5). Distances in units of $\sigma=\sigma_{1}$.

\begin{tabular}{lrcc}
\hline \hline$i$ & $\lambda_{i} / \epsilon$ & $v_{i} / \epsilon$ & $\sigma_{i} / \sigma$ \\
\hline 1 & 1 & 0 & 1 \\
2 & 2 & 1 & $4 / 3$ \\
3 & 2 & 1 & $9 / 5$ \\
4 & -1 & 1 & $37 / 15$ \\
\hline \hline
\end{tabular}

dynamic simulations, using the HOOMD-blue package [34,35], at constant temperature $(N V T)$ and constant pressure (NPT) in a three-dimensional system composed of 2048 particles in a cubic box with periodic boundary conditions, interacting through a continuous pair potential obtained by the addition of three different Fermi-Dirac distributions,

$$
U=\epsilon \sum_{i=1}^{4} \frac{\lambda_{i}}{v_{i}+\exp \left(\frac{r-\sigma_{i}}{\omega}\right)},
$$

with $\omega=0.08 \sigma$ and the other coefficients given in Table I.

The potential illustrated as the smooth curve in Fig. 1 was chosen to mimic the three length scales and energies scales employed in the one-dimensional case. The three length scales are identified by the change of the slope in the force [4]. The accessibility of the three length scales and the presence of the density anomalous region in the stable region of the pressure-temperature phase diagram is obtained by making the forces smooth and positive [36]. Units are reduced as in the 1D case, except for pressure and particle density, which are reduced as $P^{*}=P \sigma^{3} / \epsilon$ and $\rho^{*}=\sigma^{3} \rho$.

The pressure versus temperature phase diagram is shown in Fig. 4. At low temperatures, the system presents gas, LDL, HDL, and VHDL phases. Gray lines are the isochores $\rho^{*}=0.12-0.40$ and solid circles indicate gas-LDL, LDLHDL, and HDL-VHDL critical points. Similarly to what happens in the 1D case, in the vicinity of both LDL-HDL and HDL-VHDL critical points, there are TMD lines located in the stable region of the pressure-temperature phase diagram.

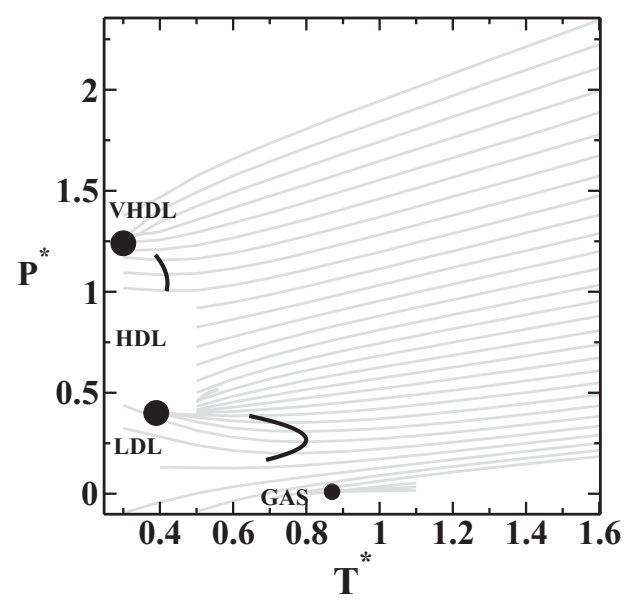

FIG. 4. Pressure vs temperature phase diagram for the 3D case. The lighter lines are isochores for $\rho^{*}=0.12-0.40$, the solid lines the TMD lines, and solid circles are the (estimated) location of the critical points. 


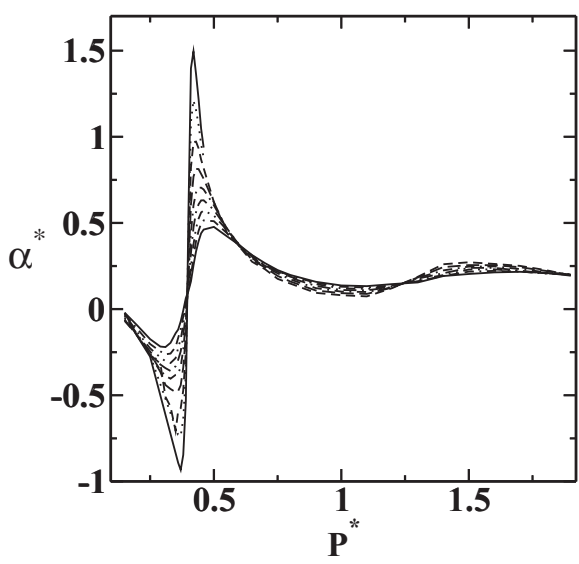

FIG. 5. Thermal expansion coefficient vs pressure for $T^{*}=$ $0.48-0.68$.

In both cases the TMD lines seem to approach the critical pressures [37], allowing us to test if the oscillatory behavior observed in $\alpha$ in the one-dimensional case can also occur in the 3D case.

This behavior is observed in Fig. 5, where the thermal expansion coefficient is drawn as a function of the pressure for different fixed temperatures $T^{*}=0.48-0.68$ in the vicinity of the LDL-HDL critical point. As in the 1D case, $\alpha$ goes to $-\infty$ as $P \rightarrow P_{c}^{-}$while it diverges to $+\infty$ as $P \rightarrow P_{c}^{+}$at the critical temperature. Since the LDL-HDL critical point occurs at a reasonable large temperature, the oscillatory behavior is quite pronounced. The large increase of $\alpha$ vs $P^{*}$ around $P^{*}=P_{c}^{*} \approx$ 0.5 is a signature of the presence of criticality, while the change in sign shows the existence of the TMD. Since the oscillation happens above the critical temperature, it is possible to identify the criticality even if the critical temperature would not be accessible. In the case of the thermal expansion coefficient in the vicinity of the VHDL-HDL critical point, the oscillations are more subtle. This behavior would be more visible if the coefficient would be computed in close vicinity of the critical point, which is more challenging given that the HDL-VHDL critical point occurs at much lower temperatures where the simulations are more costly.

\section{CONCLUSIONS}

In this paper we used an exactly solvable one-dimensional lattice model and molecular dynamic simulations of a threedimensional fluid model to show that the three length scale core-softened potential can be designed to exhibit three liquid phases (LDL, HDL and VHDL), two liquid-liquid critical points and two density anomalous regions. In addition, we propose that the oscillatory behavior observed in the thermal expansion coefficient close to criticality can be considered as a signature of the connection between a liquid-liquid critical point and a region of anomalous density increase, in the studied models. In the particular case of water, the hypothesis of a liquid-liquid critical point is still under debate due to the impossibility to experimentally probe the system in its expected location, at temperatures below the homogeneous nucleation temperature. We also propose that measurements of $\alpha$ as a function of pressure could be performed at temperatures well above criticality, where the system can be accessed (even though metastable), and used as a tool to test for the presence of a metastable second critical point.

\section{ACKNOWLEDGMENTS}

This work is partially supported by CNPq, Capes, INCTFCx, and Universidade Federal de Santa Catarina. We would like to thank C. E. Fiore and J. Nunes da Silva for useful discussions.
[1] P. C. Hemmer and G. Stell, Phys. Rev. Lett. 24, 1284 (1970).

[2] G. Stell and P. C. Hemmer, J. Chem. Phys. 56, 4274 (1972).

[3] E. A. Jagla, Phys. Rev. E 58, 1478 (1998).

[4] P. G. Debenedetti, V. S. Raghavan, and S. S. Borick, J. Chem. Phys. 95, 4540 (1991).

[5] H. Thurn and J. Ruska, J. Non-Cryst. Solids 22, 331 (1976).

[6] CRC Handbook of Chemistry and Physics, edited by R. C. Weast, 65th ed. (CRC Press, Boca Raton, FL, 1984).

[7] G. E. Sauer and L. B. Borst, Science 158, 1567 (1967).

[8] S. J. Kennedy and J. C. Wheeler, J. Chem. Phys. 78, 1523 (1983).

[9] G. S. Kell, J. Chem. Eng. Data 20, 97 (1975).

[10] T. Tsuchiya, J. Phys. Soc. Jpn. 60, 227 (1991).

[11] C. A. Angell, R. D. Bressel, M. Hemmatti, E. J. Sare, and J. C. Tucker, Phys. Chem. Chem. Phys. 2, 1559 (2000).

[12] P. H. Poole, M. Hemmati, and C. A. Angell, Phys. Rev. Lett. 79, 2281 (1997).

[13] S. Sastry and C. A. Angell, Nat. Mater. 2, 739 (2003).

[14] Y. Katayama, T. Mizutani, W. Utsumi, O. Shimomura, M. Yamakata, and K. Funakoshi, Nature (London) 403, 170 (2000).
[15] I. Saika-Voivod, F. Sciortino, and P. H. Poole, Phys. Rev. E 63, 011202 (2000).

[16] P. H. Poole, F. Sciortino, U. Essmann, and H. E. Stanley, Nature (London) 360, 324 (1992).

[17] L. Xu, P. Kumar, S. V. Buldyrev, S.-H. Chen, P. Poole, F. Sciortino, and H. E. Stanley, Proc. Natl. Acad. Sci. USA 102, 16558 (2005).

[18] O. Mishima and Y. Suzuki, Nature (London) 419, 599 (2002).

[19] D. T. Bowron, J. L. Finney, A. Hallbrucker, I. Kohl, T. Loerting, E. Mayer, and A. K. Soper, J. Chem. Phys. 125, 194502 (2006).

[20] W. P. Krekelberg, T. Kumar, J. Mittal, J. R. Errington, and T. M. Truskett, Phys. Rev. E 79, 031203 (2009).

[21] J. C. Pàmies, A. Cacciuto, and D. Frenkel, J. Chem. Phys. 131, 044514 (2009).

[22] Z. Yan, S. V. Buldyrev, N. Giovambattista, and H. E. Stanley, Phys. Rev. Lett. 95, 130604 (2005).

[23] G. Franzese, G. Malescio, A. Skibinsky, S. V. Buldyrev, and H. E. Stanley, Nature (London) 409, 692 (2001).

[24] A. B. de Oliveira, G. Franzese, P. A. Netz, and M. C. Barbosa, J. Chem. Phys. 128, 064901 (2008). 
[25] D. Y. Fomin, N. V. Gribova, V. N. Ryzhov, S. M. Stishov, and D. Frenkel, J. Chem. Phys. 129, 064512 (2008).

[26] J. R. Errington and P. G. Debenedetti, Nature (London) 409, 318 (2001).

[27] J. R. Errington, T. M. Truskett, and J. Mittal, J. Chem. Phys. 125, 244502 (2006).

[28] Z. Yan, S. V. Buldyrev, N. Giovambattista, P. G. Debenedetti, and H. E. Stanley, Phys. Rev. E 73, 051204 (2006).

[29] A. B. de Oliveira, P. A. Netz, and M. C. Barbosa, Europhys. Lett. 85, 36001 (2009).

[30] S. V. Buldyrev and H. E. Stanley, Physica A 330, 124 (2003).

[31] H. Takahasi and L. Van Hove, Near Critical Behavior of Aqueous Systems (Academic, New York, 1966), p. 1.
[32] M. A. A. Barbosa, F. V. Barbosa, and F. A. Oliveira, J. Chem. Phys. 134, 024511 (2011).

[33] C. E. Fiore and M. G. E. da Luz, Phys. Rev. Lett. 107, 230601 (2011).

[34] J. A. Anderson, C. D. Lorenz, and A. Travesset, J. Comput. Phys. 227, 5342 (2008).

[35] HOOMD-blue, [http://codeblue.umich.edu/hoomd-blue/index. html], December 2011.

[36] N. M. Barraz, Jr., E. Salcedo, and M. C. Barbosa, J. Chem. Phys. 135, 104507 (2011).

[37] E. Salcedo, A. B. de Oliveira, N. M. Barraz, Jr., C. Chakravarty, and M. C. Barbosa, J. Chem. Phys. 135, 044517 (2011). 